\title{
A virtual pipe rig for testing acoustic leak detection correlators: Proof of concept
}

\author{
M.J. Brennan ${ }^{\text {a,* }}$, F. Kroll de Lima ${ }^{\text {a }}$, F.C.L. de Almeida ${ }^{\text {b }}$, P.F. Joseph ${ }^{c}$, A.T. Paschoalini ${ }^{\text {a }}$ \\ ${ }^{a}$ Department of Mechanical Engineering, UNESP, Ilha Solteira, São Paulo 15385-000, Brazil \\ ${ }^{\mathrm{b}}$ Department of Biosystem Engineering, UNESP, Tupã, São Paulo 17602-496, Brazil \\ ${ }^{c}$ Institute of Sound and Vibration Research, University of Southampton, SO17 1BJ, UK
}

\section{A R T I C L E I N F O}

\section{Article history:}

Received 10 April 2015

Received in revised form 17 September 2015

Accepted 21 September 2015

Available online 17 October 2015

\section{Keywords:}

Water leaks

Correlation

Pipe vibration

\begin{abstract}
A B S T R A C T
Acoustic correlators have been used for many years to locate and detect leaks in buried water distribution pipes. Currently, the only way to compare different correlators directly is in the field. This can be problematic as it may be difficult to present exactly the same conditions to each correlator. In recent years, the way in which leak noise propagates in buried water pipes has been determined, and this has enabled the development of a virtual pipe whose behaviour can be simulated in the computer. By coupling the filtering properties of the pipe with electrodynamic shakers, a proof-of-concept virtual pipe test-rig has been developed that will allow different correlators to be compared directly in laboratory conditions. Different situations, such as pipe material and size, and measurement positions, as well as leak strength can be simulated. The theoretical basis of the test-rig and details of the proof-of-concept system are discussed in this paper. It is shown that careful consideration of the dynamics of the shakers is vital if the system is to faithfully model situations that are found in the field.
\end{abstract}

(c) 2015 Elsevier Ltd. All rights reserved.

\section{Introduction}

Leakage from buried water distribution pipelines is a global problem [1]. Acoustic correlators have been used for more than 30 years to detect and locate leaks from water pipes [2]. Although they work well for metallic pipes there are some issues with plastic pipes $[3,4]$. Waves in these pipes suffer from much higher rates of attenuation $[3,4]$, which makes the problem of water leak detection more challenging. The emphasis in this paper is, therefore, on plastic pipes. In most cases accelerometers (or less commonly, hydrophones) are located at convenient access points either side of a suspected leak to sense the noise generated by the leak. The peak in the correlation function between the signals from these sensors is used to determine the difference in propagation times between the leak and the sensors. By combining this with knowledge of the wave speed, the location of the leak can be determined.

Water companies can choose different correlators from different manufacturers, but it is not an easy task for them to know a-priori which is the most effective for their pipe network. Currently, the only way to compare acoustic correlators directly is to conduct a field test. This can be problematic as it may be

\footnotetext{
* Corresponding author.

E-mail address: mjbrennan0@btinternet.com (M.J. Brennan).
}

difficult to present identical conditions to each correlator. Another issue relating to the effective use of correlators concerns the training of personnel in their use. Ideally, the effective use of correlators requires the user to be exposed to, and gain experience with, a diverse range of pipe types and leak strengths and types. Both of these issues motivate the need to develop a bench-top test-rig that can replicate the pipe vibrations generated by a leak in the field, a so-called "virtual pipe test-rig". To achieve this, a thorough understanding of the way in which a leak generates noise and the way in which that noise propagates through a buried pipe is necessary. The research programme conducted by the authors and colleagues over the past ten years or so has helped to gain this understanding, for example [5-12]. This work covers the central understanding needed to develop a virtual rig, namely the development of a physical model for wave propagation along the plastic pipe [6], the incorporation of the physical model with a signal processing model [8], and the effects of sensors on the cross-correlation coefficient [9].

In particular, it has been found that in buried plastic water pipes, leak noise mainly propagates at low frequencies, below about $200 \mathrm{~Hz}$, because of the high levels of damping in the pipe-wall, and the radial motion of the pipe-wall due to the coupling between the fluid and the pipe. The wave responsible for propagating the noise is a low-frequency, non-dispersive, axisymmetric, coupled 
structural-fluid wave located well below the ring frequency [6,7]. The key to the development of the test-rig is this phenomenon, and the ability to couple the analytical pipe model with the cross-correlation coefficient [8] and an accelerometer, which measures pipe vibration that is strongly correlated to the leak noise [9]. Experimental results reported in [12] validate much of the previous work, and the data from that paper is used here to check the veracity of the virtual pipe test-rig.

The aim of this paper is to describe the theoretical basis behind a virtual pipe test-rig, and to describe the tests conducted on a proof-of-concept device. The paper is organised as follows. In Section 2, a brief overview of leak detection using cross-correlation is given. This is followed by the theoretical basis of the virtual-pipe test rig in Section 3. Section 4 describes the implementation of a proof-of-concept virtual-pipe test rig, and Section 5 discusses the importance of choosing a shaker with the appropriate dynamics for such a system. Finally, some conclusions are given in Section 6 .

\section{Overview of leak detection using acoustic correlation}

Fig. 1 shows a typical leak detection problem, in which a leak occurs at an unknown position in a buried water pipe. The leak generates broadband noise, which propagates along the pipe to acoustic or vibration sensors that are located at convenient access points either side of the leak. The difference in the arrival times of the noise at the sensors (time delay) is used to determine the position of the leak, which is given by [8],

$d_{2}=\frac{d-c T_{0}}{2}$

where $c$ is the speed of propagation of the leak noise, $d$ is the distance between the sensors, and $T_{0}=\left(d_{1}-d_{2}\right) / c$ is the time delay estimate. In plastic pipes the noise propagates in a predominantly fluid-wave that is strongly coupled to the pipe-wall $[6,7]$. Because of this, the wave (leak noise) can be measured by sensing the vibration of the pipe-wall or a valve using accelerometers, or by using a hydrophone inserted into the pipe [12]. In many cases the wavespeed is estimated from tables, but it can also be measured insitu [13]. The time delay $T_{0}$ is estimated from the peak in the cross-correlation function between the two measured signals $x_{1}(t)$ and $x_{2}(t)$, which is given by [8]

$R_{x_{1} x_{2}}(\tau)=\frac{1}{2 \pi} \int_{-\infty}^{+\infty} S_{x_{1} x_{2}}(\omega) e^{j \omega \tau} d \omega$,

where $j=\sqrt{-1}$, and $S_{x_{1} x_{2}}(\omega)=\left|S_{x_{1} x_{2}}(\omega)\right| e^{j \phi}$ is the cross-spectral density (CSD) function, in which $\left|S_{x_{1} x_{2}}(\omega)\right|$ is the modulus and $\phi$ is the phase. If there is a pure time delay $T_{0}$ between the two signals then the modulus is a constant and $\phi=-\omega T_{0}$. Rather than use the crosscorrelation function, it is preferable to use the cross-correlation coefficient, which is given by $\rho(\tau)=R_{x_{1} x_{2}}(\tau) / \sqrt{R_{x_{1} x_{1}}(0) R_{x_{2} x_{2}}(0)}$, which has a value between -1 to $+1 ; R_{x_{1} x_{1}}(0)$ and $R_{x_{2} x_{2}}(0)$ are the auto-correlation functions of the signals when $\tau=0$ at positions 1 and 2 respectively

Typical processed leak signals are illustrated from measurements made on a buried plastic pipe rig in the UK using accelerometers [13]. The test rig is shown Fig. 2. It consists of a $120 \mathrm{~m}$ long, high performance polyethylene (HPPE) pipe, buried at a depth of about $0.8 \mathrm{~m}$ in an open field. The pipe has a Young's modulus of about $2 \times 10^{9} \mathrm{Nm}^{-2}$, an outer radius of $80 \mathrm{~mm}$ and a wall thickness of $9.85 \mathrm{~mm}$. The loss factor was found to be 0.1 [14]. Properties of the test rig are shown in Table 1. The processed data are shown in Fig. 3 [13]. Fig. 3(a) shows the modulus of the CSD normalised by its maximum value, and Fig. 3(b) shows the phase. Also shown in Fig. 3(b) is a straight line corresponding to $\phi=-\omega T_{0}$. The coherence is shown in Fig. 3(c), where it can be seen that the bandwidth over which there is potentially time delay information is about $20-120 \mathrm{~Hz}$ corresponding to the frequency range at which the coherence is not close to zero. Finally, Fig. 3(d) shows the cross-correlation coefficient in which the time delay is indicated. This figure is the one most often used in acoustic correlators to indicate the presence of a leak. The time delay can be determined from the peak in the cross-correlation function. It can also be determined from the phase of the CSD, because the gradient of the weighted least squares straight line fit to the phase is equal to the time delay [11]. This can then be used in Eq. (1) to determine the location of the leak, if the wave-speed $c$ is also known. It is clear from Fig. 3 that the signal characteristics measured on buried plastic pipes necessary for leak detection are complex and it is these that need to be simulated on a laboratory virtual rig.

\section{Development of the virtual pipe test-rig}

\subsection{Model of water pipe and acoustic correlation}

To develop and build a virtual pipe test-rig, a model of leak noise propagation is needed. A block diagram of this model is shown in Fig. 4. The source of the noise (the leak) is assumed to be spectrally white in the frequency range of interest $(<200 \mathrm{~Hz})$ with a Power Spectral Density (PSD) of $S_{l l}$. This is considered to be a reasonable assumption for plastic pipes [15], which is the primary focus of the work reported here. The frequency response function (FRF) between the acoustic pressure at the leak location

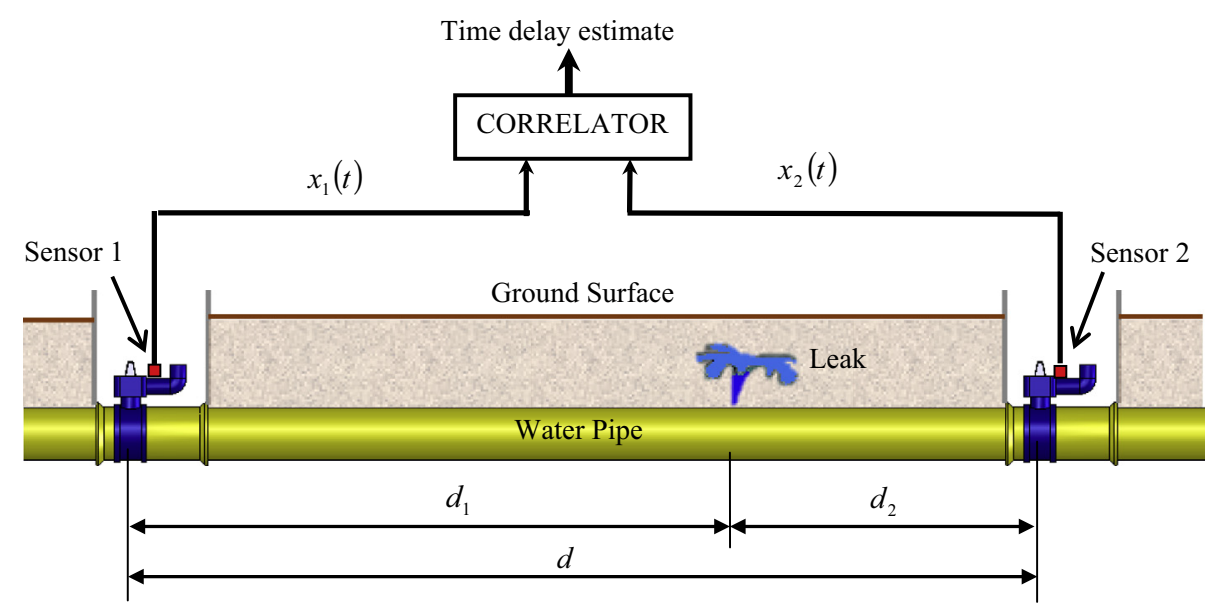

Fig. 1. Schematic of leak detection in a buried plastic water pipe using acoustic/vibration signals with a leak in between the two sensors. 
(a)

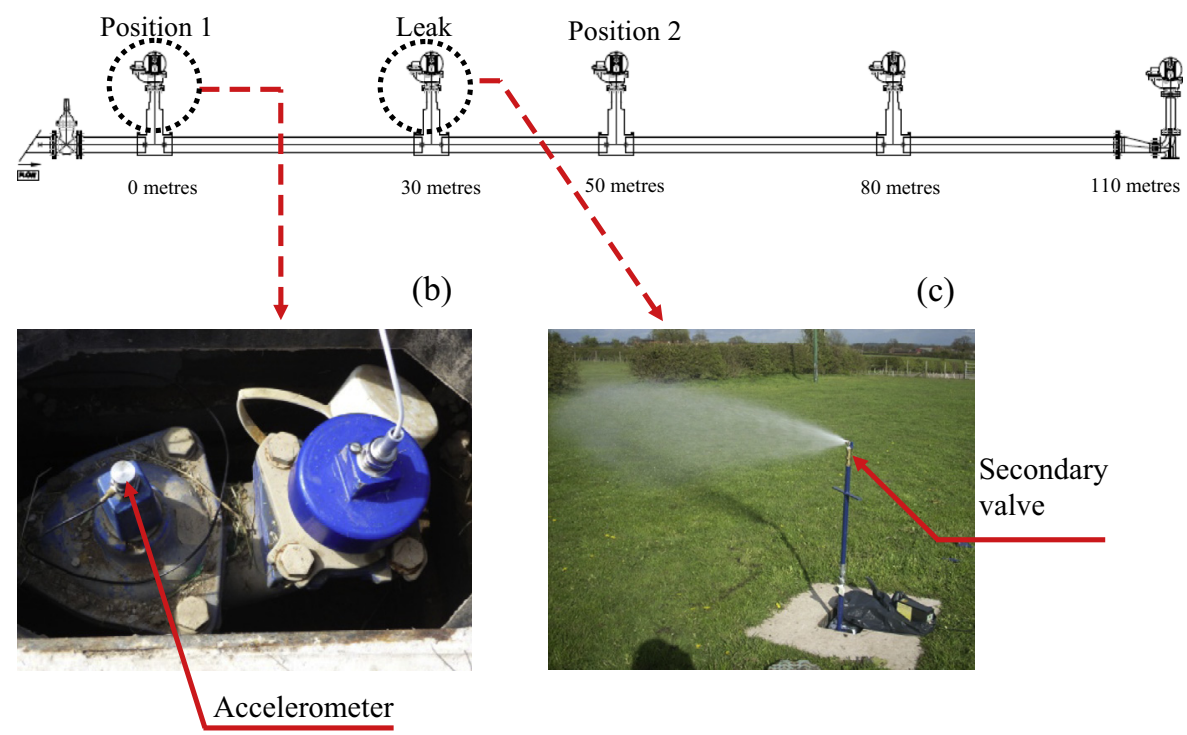

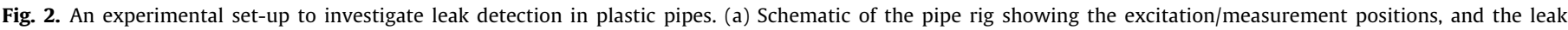
position. (b) Photograph of the instrumentation at Positions 1 and 2. (c) Photograph of the leak with the valve fully open.

Table 1

Physical properties of the pipe-model.

\begin{tabular}{ll} 
Mean radius of the pipe & $75 \mathrm{~mm}$ \\
Pipe-wall thickness & $9.85 \mathrm{~mm}$ \\
Pipe Young's modulus & $2 \times 10^{9} \mathrm{~N} / \mathrm{m}^{2}$ \\
Pipe loss factor & 0.1 \\
Bulk modulus of water & $2.2 \times 10^{9} \mathrm{~N} / \mathrm{m}^{2}$ \\
Free-field wave-speed in water & $1500 \mathrm{~m} / \mathrm{s}$ \\
$d_{1}, d_{2}$ & $30 \mathrm{~m}, 20 \mathrm{~m}$ \\
\hline
\end{tabular}

and the measurement position a distance $u$ from the leak is given by [8]

$H(\omega, u)=e^{-\omega \beta u} e^{-j \omega u / c}$

where $c=c_{f} /(1+2 B a / E h)^{1 / 2}$ is the speed of the wave responsible for leak noise propagation and $\beta=(\eta B a / E h) /\left(c_{f}(1+(2 B a / E h))^{1 / 2}\right)$ is the attenuation factor, in which $c_{f}$ and $B$ are the free-field wavespeed and bulk modulus of water respectively, and $E, a, h$ and $\eta$ are the Young's modulus, the mean pipe radius, the pipe-wall thickness, and loss factor of the pipe-wall respectively. Pipe filters 1 and 2 in Fig. 4 are simply determined from Eq. (3) by setting $u=d_{1}$ and $u=d_{2}$ respectively.

Well below the ring frequency (which is the case here), the radial displacement of the pipe at the measurement position is proportional to the acoustic pressure inside the pipe [9]. However, the radial acceleration of the pipe is of practical importance, and this is simply related to the radial displacement by $-\omega^{2}$ so the FRF relating the acoustic pressure to the radial acceleration is given by [9]

$G(\omega)=-\frac{a^{2} \omega^{2}}{E h}$

The FRFs for the sensor blocks in Fig. 4 are simply given by Eq. (4). Assuming that the sensors at positions 1 and 2 have identical characteristics, the CSD of the measured acceleration signals is given by [9]

$S_{x_{1} x_{2}}(\omega)=S_{l l}|G(\omega)|^{2} H^{*}\left(\omega, d_{1}\right) H\left(\omega, d_{2}\right)$ where * denotes the complex conjugate. Combining Eqs. (3)-(5) results in

$S_{x_{1} x_{2}}(\omega)=\frac{S_{l l} a^{4}}{(E h)^{2}} \omega^{4} e^{-\beta d \omega} e^{-j \omega T_{0}}$

which can be written in non-dimensional form as

$\hat{S}_{x_{1} x_{2}}(\hat{\omega})=\frac{S_{x_{1} x_{2}}(\omega)}{\left(\frac{S_{l l} a^{4}}{(E h)^{2}(\beta d)^{4}}\right)}=\hat{\omega}^{4} e^{-\hat{\omega}} e^{-j \hat{\omega} \hat{T}_{0}}$

where $\hat{\omega}=\omega \beta d$ and $\hat{T}_{0}=T_{0} /(\beta d)$. The modulus and the phase of the normalised cross spectrum is plotted in Fig. 5(a) and (b) respectively. The phase is simply a straight line with a slope of $-\hat{T}_{0}$, but the modulus $\left|\hat{S}_{x_{1} x_{2}}(\hat{\omega})\right|=\hat{\omega}^{4} e^{-\hat{\omega}}$, is frequency dependent, which is important, as this has a profound effect on the shape of the crosscorrelation function [9]. The frequency sensitivity of the accelerometer means that it acts as a high-pass filter of the leak noise, while the pipe acts as a low-pass filter. Together they combine to create a band-pass filter, which is evident in Fig. 5(a). By setting the derivative of Eq. (6b) with respect to frequency to zero, it is found that the modulus of the cross-spectrum peaks when $\omega=4 /(\beta d)$. Thus, the frequency band over which there is measured leak noise energy is governed only by the product $\beta d$. If the distance between the two sensors is large and the damping in the pipe is high, then the leak noise energy, as measured by the accelerometers, contains only low frequencies. If geophones are used instead of accelerometers then the cross-spectrum peaks at $\omega=2 /(\beta d)$, i.e. half the frequency compared to when accelerometers are used. This occurs because there is a factor of $j \omega$ rather than $-\omega^{2}$ in the FRF relating the acoustic pressure to the geophone output (velocity rather than acceleration), in Eq. (4). This means that the low pass-filtering effect of the pipe has a greater effect on the system behaviour and the peak in the cross-spectrum is shifted to a lower frequency.

By combining Eqs. (2) and (6a), the cross-correlation function, and hence the time delay, can be estimated. This model has been validated previously with experimental data in $[8,9]$. Note that in practice the radial acceleration of the pipe is not generally measured directly. Accelerometers are attached to the pipe system at a convenient access point, which in many cases is a fire hydrant. 

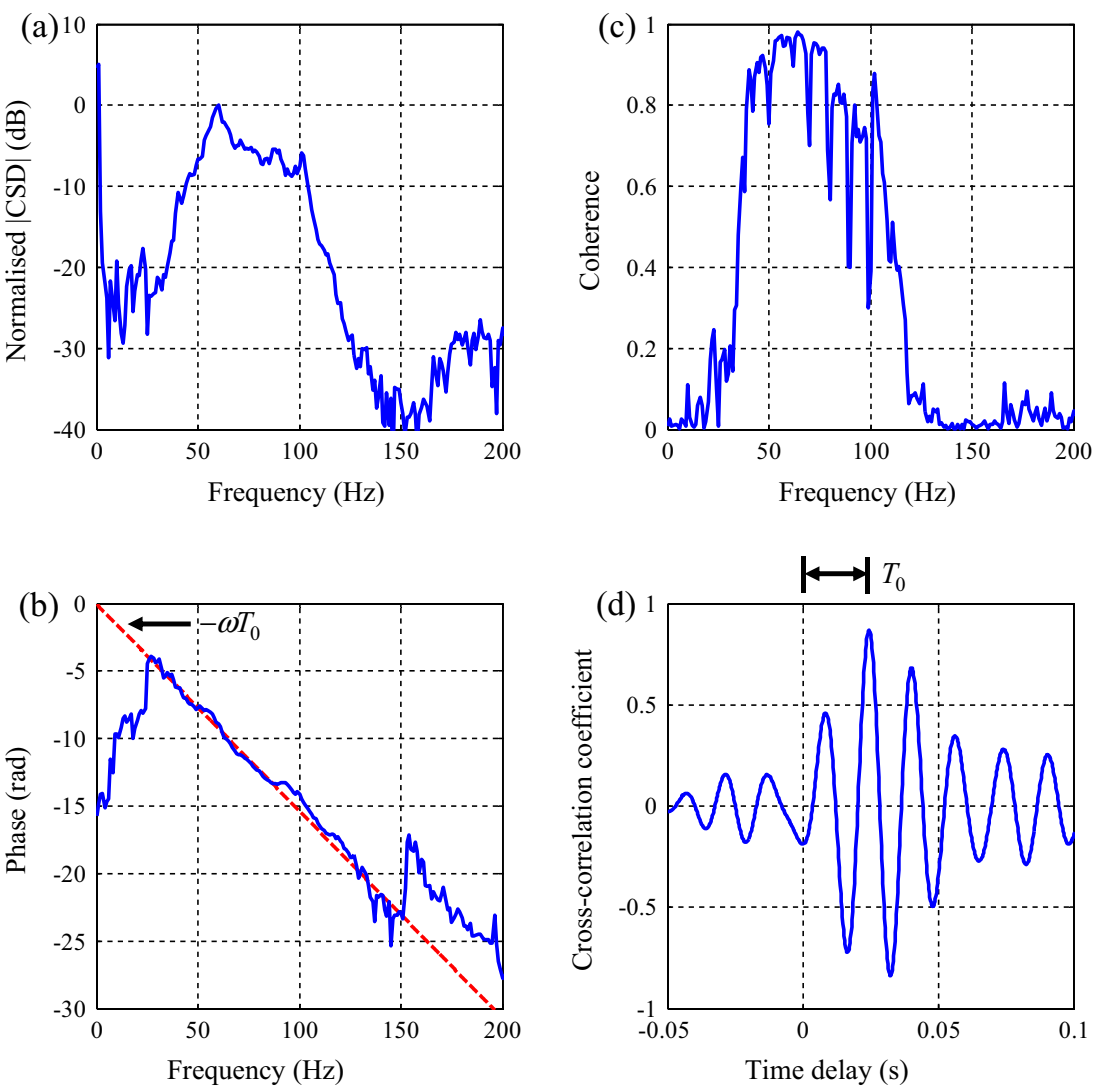

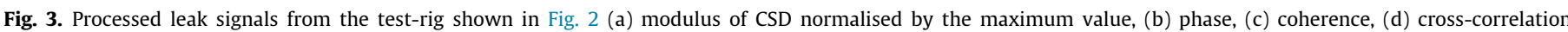
coefficient.

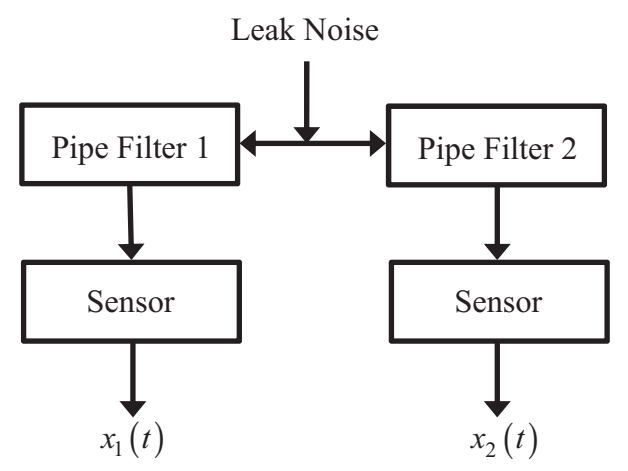

Fig. 4. Model of the system shown in Fig. 1.

However, it has been found that this acceleration is strongly correlated to the related acceleration of the pipe-wall, which in turn is related to the acoustic pressure in the pipe by Eq. (5) $[7,12]$.

\subsection{Design of the virtual pipe test-rig}

A block diagram of the virtual pipe test-rig is shown in Fig. 6 . The model of the pipe (without the sensors) with a source of random noise to simulate the leak is implemented in a laptop computer using Matlab ${ }^{\circledR}$. To generate vibration that replicates the radial vibration of the pipe at the sensor positions, two shakers and power amplifiers are required, which are driven by signals that are output from the pipe model. However, filters have to be used to compensate for the dynamics of the power amplifiers (and the shakers if possible), so that they will have a minimal effect on the signals measured by the acoustic correlators. If the two shaker and power amplifier sets have FRFs of $C_{1}(\omega)$ and $C_{2}(\omega)$ then Eq. (5) is modified by the multiplication of $C_{1}^{*}(\omega) C_{2}(\omega)$.

\section{Proof-of-concept system}

To determine whether a virtual pipe test-rig could simulate the behaviour of the system shown in Fig. 1, the design described in the previous section was implemented using a laptop computer, and two small modified loudspeakers that had integral power amplifiers were used as shakers. It is recognised that more robust vibration exciters would be necessary in a practical system, but the small loudspeakers were considered to be adequate for a proof-ofconcept study. The pipe model was interfaced with the sound card to generate the signals to drive the loudspeakers.

Prior to carrying out experiments with the complete test rig, the FRFs of the loudspeakers together with the power amplifiers were measured. They were excited with random noise generated using a laptop and the soundcard, and the responses were measured using PCB type 352A25 accelerometers. One of the loudspeakers and an accelerometer are shown in Fig. 7(a). It was found that the power amplifiers have second order high-pass filters incorporated, and these were each compensated for in the test-rig by dividing by $-\omega^{2}$. The displacement response of each loudspeaker was calculated by twice integrating the accelerometer signals to give the FRF of displacement/input voltage. The magnitude and phase of these FRFs are in Fig. 7(b) and (c). It can be seen that there are differences between each loudspeaker, with the response being relatively flat between about $20 \mathrm{~Hz}$ and $80 \mathrm{~Hz}$, falling off rapidly above the resonance at about $90 \mathrm{~Hz}$. Within the frequency range $20 \mathrm{~Hz}$ and $80 \mathrm{~Hz}$, the phase response is broadly similar in each 

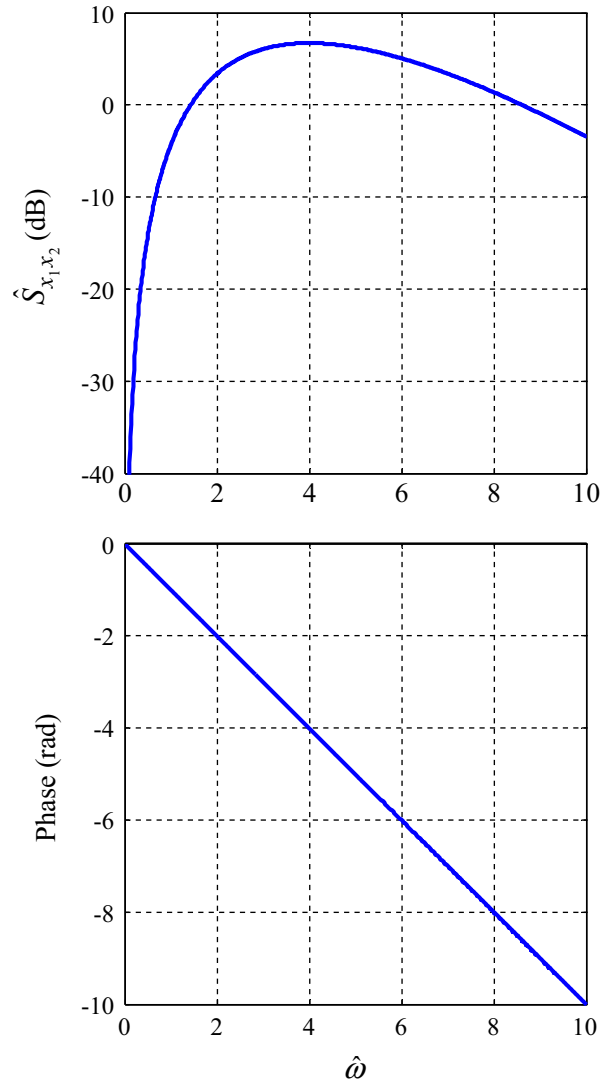

Fig. 5. Normalised CSD of the pipe model and accelerometers as a function of nondimensional frequency.

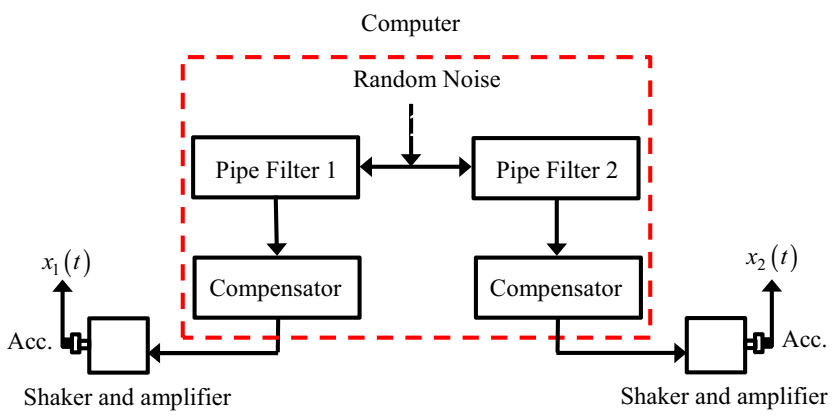

Fig. 6. Virtual pipe test-rig.

case, except for a frequency region around $60 \mathrm{~Hz}$ due to some additional dynamics in loudspeaker 2 . It should be noted that although the phase is not zero in the frequency range between $20-80 \mathrm{~Hz}$ for both loudspeakers, changing by about $\pi$ radians, the phase is similar for both devices. This is not a problem, however, as it is the phase between the devices that is important for the virtual pipe rest-rig.

As the loudspeakers were considered to have appropriate dynamics within the frequency range $20-80 \mathrm{~Hz}$ (this is discussed further in the next section), a virtual pipe test-rig was implemented within this frequency range. The subsequent data were compared with the model described in the previous section, and also with data taken from the field, which is shown in Fig. 3. The data used in the pipe model is given in Table 1, which are typical for plastic water distribution pipes in the UK and were taken from [13].
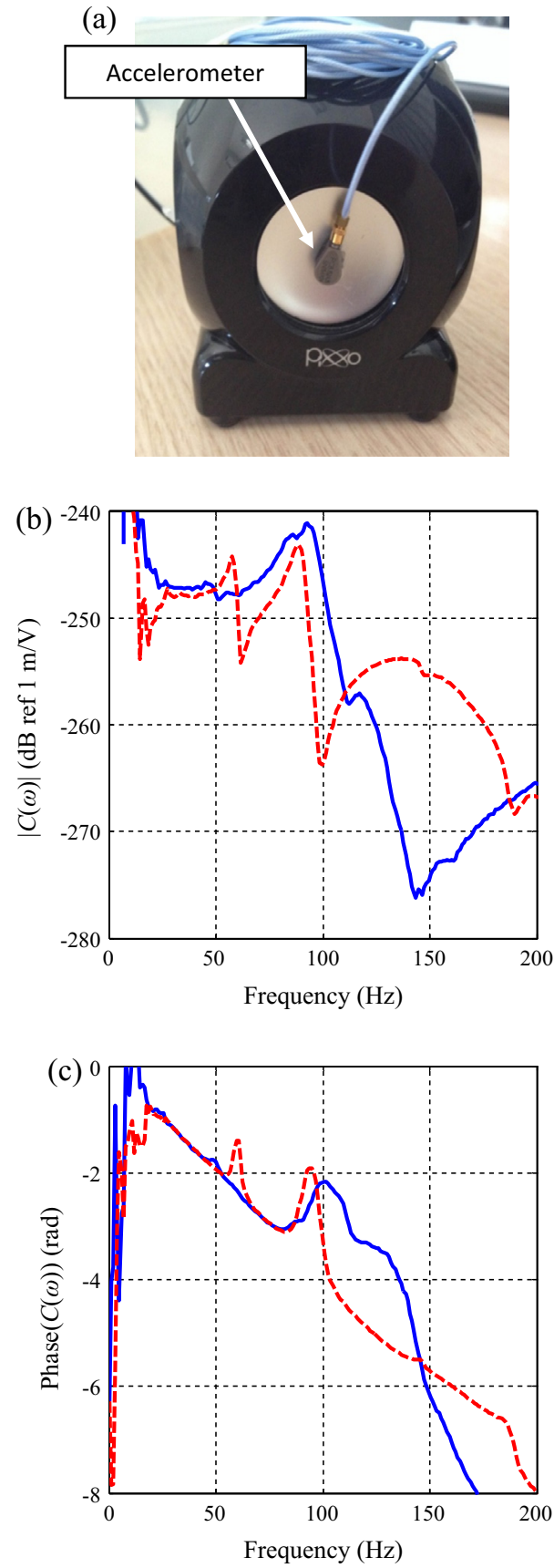

Fig. 7. Type of loudspeaker used in the virtual pipe test-rig and the dynamic characteristics of the two loudspeakers used. (a) Photograph of one of the loudspeakers, (b) magnitude of the FRF, (c) phase of the FRF; blue solid line, loudspeaker 1; red dashed line loudspeaker 2. (For interpretation of the references to colour in this figure legend, the reader is referred to the web version of this article.)

The results are shown in Fig. 8. Fig. 8(a) shows the normalised modulus of the CSD, Fig. 8(b) shows the phase and Fig. 8(c) shows the cross-correlation function. It can be seen in Fig. 8(a) that in the frequency range of $20-80 \mathrm{~Hz}$, the moduli of the CSDs for the virtual test-rig are similar to the model discussed in Section 3 and the field data shown in Fig. 3. The reason that the modulus increases with frequency is primarily due to the characteristics of the accelerometers as discussed in Section 3. Above about $80 \mathrm{~Hz}$ the responses from the virtual pipe test-rig reduce with frequency because of loudspeaker resonances and filtering effect of the pipe. The field 

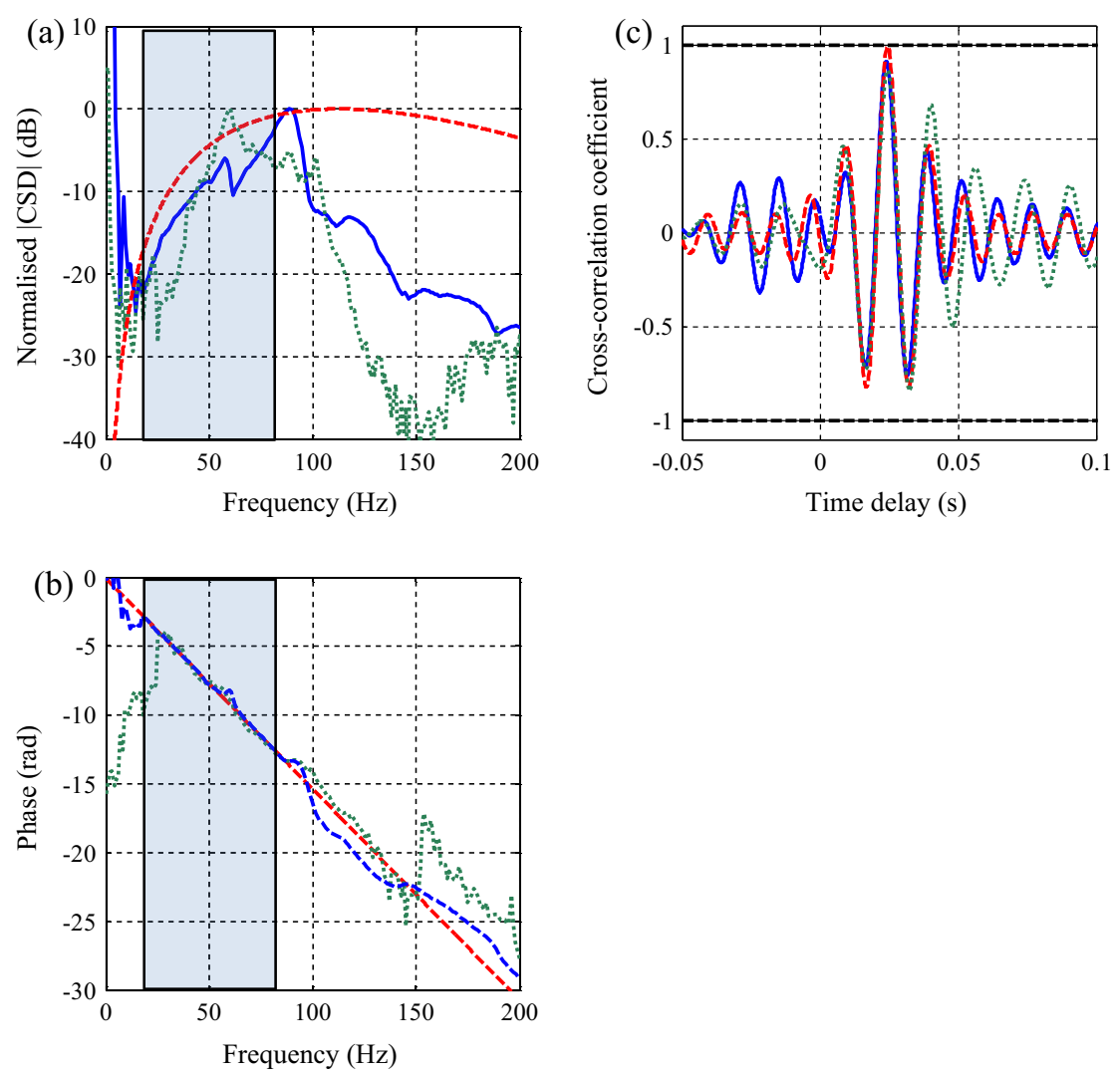

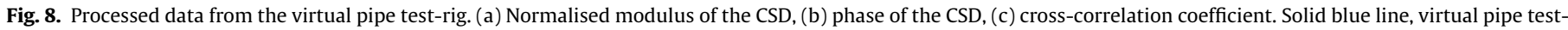

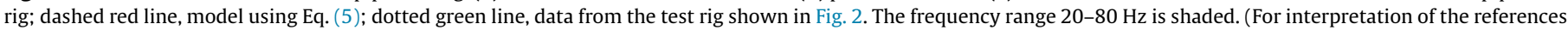
to colour in this figure legend, the reader is referred to the web version of this article.)

data also reduces with frequency about $100 \mathrm{~Hz}$, and this is due to the dynamics of the buried pipe system [13].

The phase characteristics between the three cases are also similar within the frequency range $20-80 \mathrm{~Hz}$. The similarities in the CSDs for all three cases suggests that the model and the test-rig give a reasonable representation of the field conditions within this frequency range. Finally, Fig. 8(c) shows the cross-correlation coefficients for all three cases. It can be seen that there are some differences, but close to the time delay related to the noise propagation through the pipe, the differences are relatively small. The time delays for the model and the field data are both $24.4 \mathrm{~ms}$ and for the virtual pipe test-rig it is $24 \mathrm{~ms}$, a difference of about $1.7 \%$. It should be noted that the Young's modulus of the pipe in the model was chosen so that the wavespeed of about $410 \mathrm{~m} / \mathrm{s}$ matched that measured in the field, so the small difference in time delay is due solely to the dynamics of the loudspeakers.

To demonstrate the ability of the test-rig to simulate various conditions, three representative results are shown in Fig. 9(a)(c). The configurations chosen are shown at the top of the figure. In Fig. 9(a)(i-iii), the results for the case considered previously, i.e. $d_{1}=30 \mathrm{~m}$ and $d_{2}=20 \mathrm{~m}$ are shown as a benchmark test, in Fig. 9(b)(i-iii), the same distance between the sensors is considered, but the leak is positioned such that $d_{1}=40 \mathrm{~m}$ and $d_{2}=10 \mathrm{~m}$, and finally in Fig. 9(c)(i-iii), the distance between the sensors is doubled and the leak is positioned such that $d_{1}=45 \mathrm{~m}$ and $d_{2}=55 \mathrm{~m}$. The Roman numerals (i, ii or ii) correspond to the modulus and phase of the CSD, and the cross-correlation coefficient respectively. It is clear, that in all three cases, the phase changes as expected, as does the cross-correlation function. The distances calculated from the peaks in the cross-correlation functions using the data in Table 1 (which results in a wavespeed of $410 \mathrm{~m} / \mathrm{s}$ ) are $d_{1}=29.92 \mathrm{~m}, 39.85 \mathrm{~m}, 45.02 \mathrm{~m}$ for the cases in Fig. 9(a), (b), and (c) respectively. The percentage difference between the actual and the estimated difference is less than $0.5 \%$ in all cases. The other feature, which is not so obvious in the results of the virtual test-rig, is that the shape of the modulus of the cross-spectrum, which is an indication of the filtering properties of the pipe-sensor system, is a function of the total distance between the sensors and not the position of the leak between the sensors, as seen in Fig. 9(a)-(c)(i). As the distance between the sensors is increased, the low-pass filtering effect of the pipe increasingly dominates over the high-pass filtering effect of the sensors, resulting in a shift the peak in the modulus of the cross-spectrum to lower frequencies. This effect can be seen by comparing Fig. 9(c)(iii) with Fig. 9(a)(i, ii).

\section{Discussion}

In the last section, it was shown that the bandwidth over which the proof-of-concept virtual pipe test rig was effective, was limited. In the field, the low frequency limit is governed by background noise and limitations in the low frequency response of the sensors. In the virtual pipe test-rig the background noise is not an issue as this can be controlled in laboratory conditions, but the accelerometer sensitivity could possibly be an issue. However, piezoelectric sensors were used in this work and these operate effectively above about $20 \mathrm{~Hz}$, which was the low frequency limit for the field data. Thus, the low frequency limit is deemed to be achievable by the test rig, and is not likely to be a problem in any future development. 

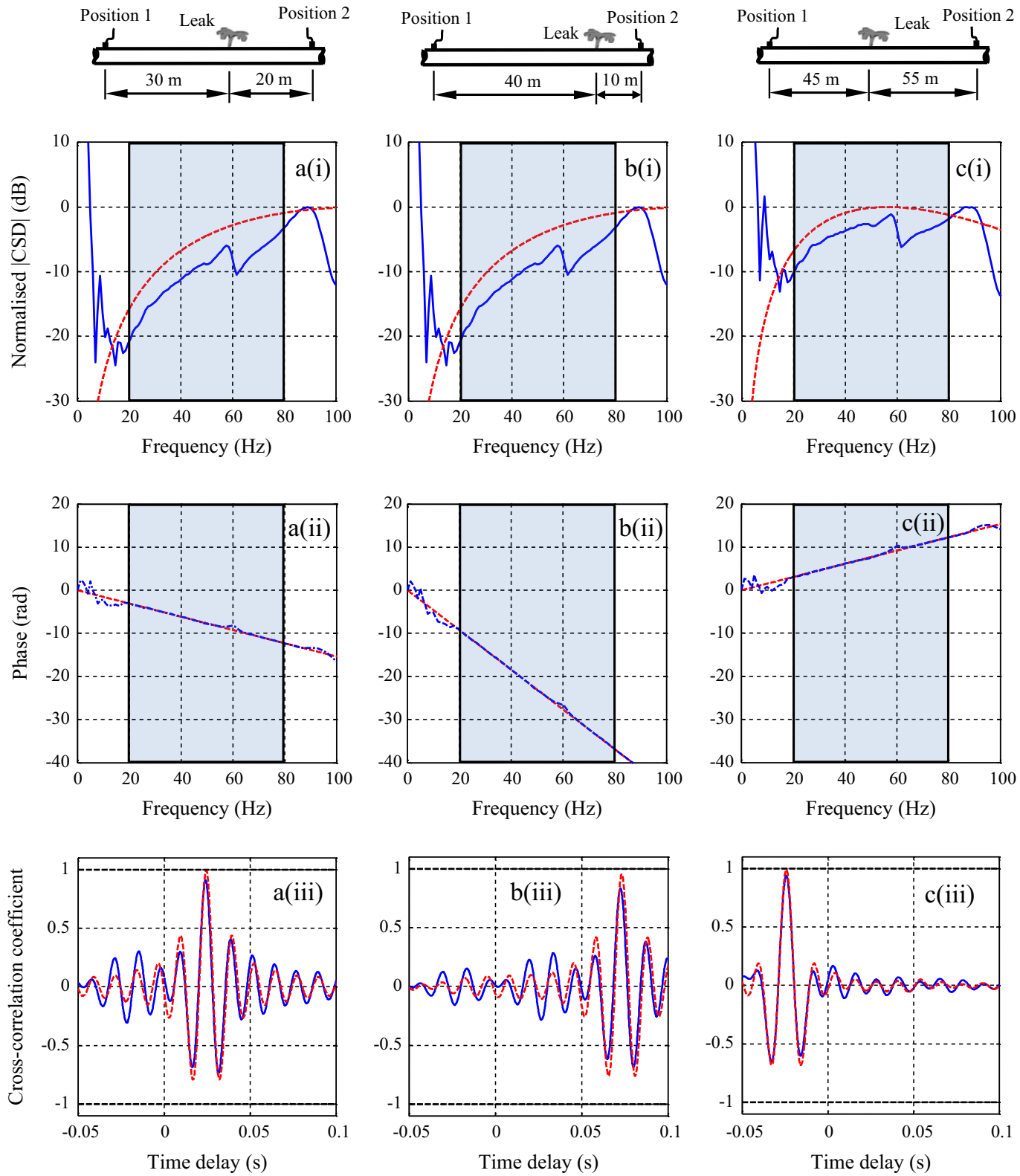

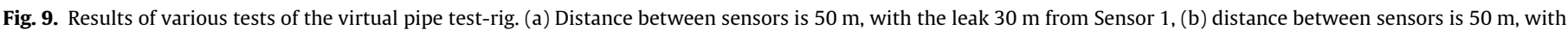

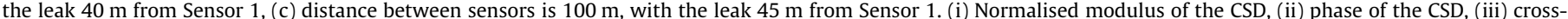

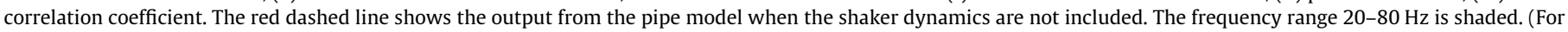
interpretation of the references to colour in this figure legend, the reader is referred to the web version of this article.)

The high frequency limit, however, could be problematic and deserves closer analysis. Examining Fig. 8(a), it can be seen that the resonance frequencies of the shakers govern the high frequency limit. It can be seen that above this frequency the behaviour of the virtual pipe test-rig does not correspond to the ideal model described in Section 3. The reason for this is that the displacement of a shaker is no longer proportional to the input current above the resonance frequency. This is because each shaker acts as a second order low-pass filter. To illustrate the effects of shaker dynamics on the CSD and the cross-correlation function some simulations are carried out. Each shaker is modelled as a classical second-order system with FRF given by

$H(\omega)=\frac{\omega_{n}^{2}}{\omega_{n}^{2}-\omega^{2}+j 2 \zeta \omega \omega_{n}}$ where $\omega_{n}$ is the natural frequency of the shaker and $\zeta$ is the damping ratio. To allow for shakers with different characteristics, shaker 1 is deemed to have an FRF of $H_{1}(\omega)$ and shaker 2 an FRF of $H_{2}(\omega)$. Eq. (6a) can be modified to account for the shakers by the multiplication of $H_{1}^{*}(\omega) H_{2}(\omega)$. Three situations are considered for the benchmark test case illustrated in Fig. 8: the natural frequency of one of the shakers is set to, (a) $20 \mathrm{~Hz}$, (b) $50 \mathrm{~Hz}$ and (c) $80 \mathrm{~Hz}$, and the natural frequency of the other shaker is set to be $10 \%$ higher in each case. The damping ratio of both shakers is set to 0.1 . The results are shown in Fig. 10. The letters ( $a, b$ and $c)$ correspond respectively to the cases above, and the Roman numerals (i, ii or ii) correspond to the modulus and phase of the CSD, and the cross-correlation coefficient respectively. The effect the shaker dynamics is clear to see, especially on the modulus of the CSD and the cross-correlation coefficient. Effectively two narrow band 

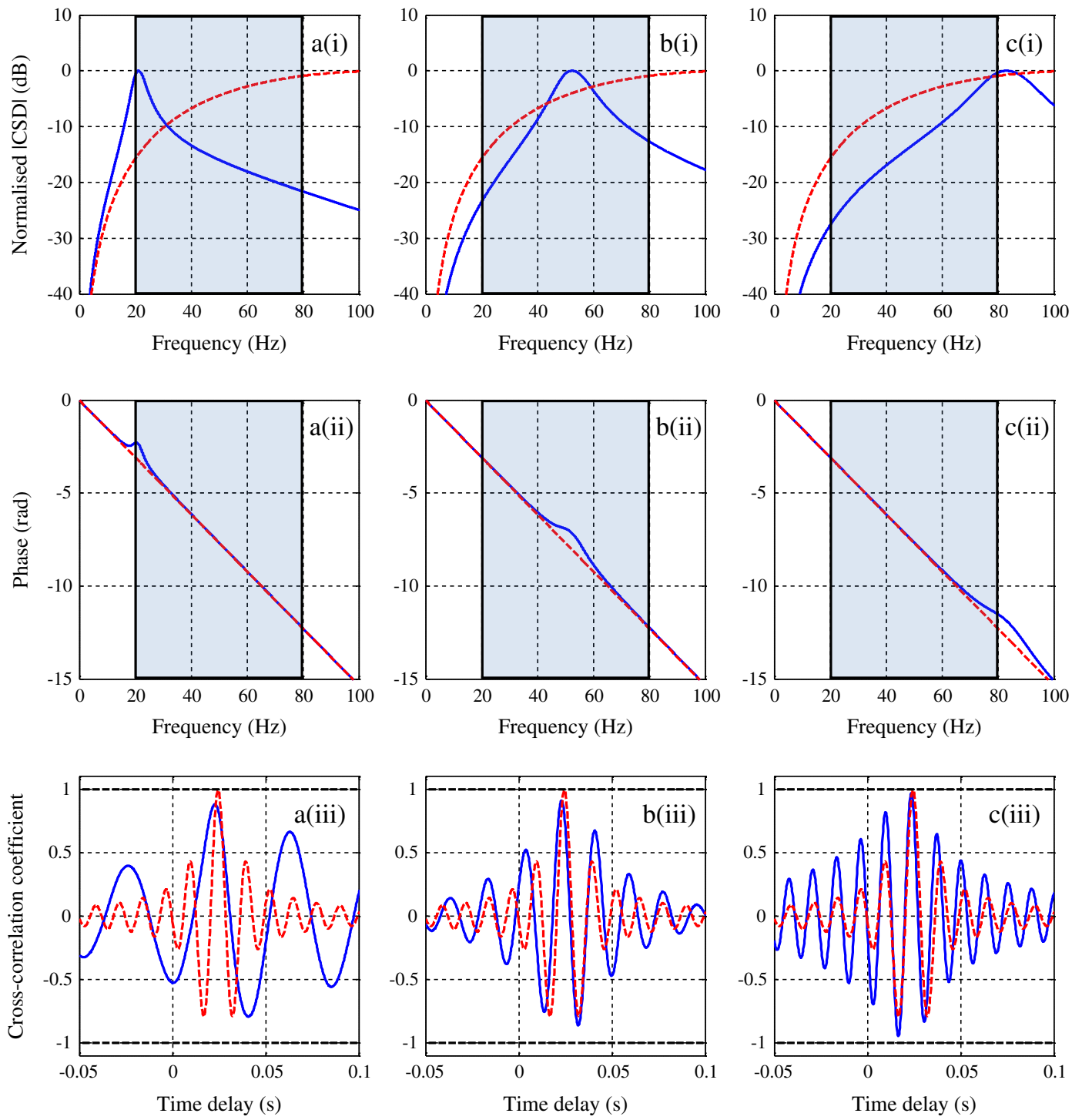

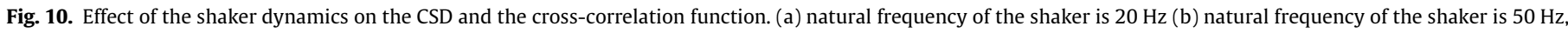

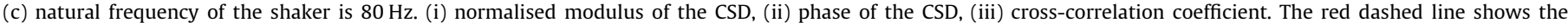

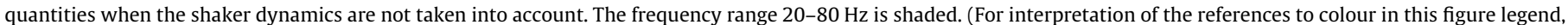
the reader is referred to the web version of this article.)

filters have been added in addition to those related to the pipe-sections and the accelerometers, further reducing the bandwidth over which the analysis is carried out. The effect of this is particularly evident in the cross-correlation coefficient. The deviation in the phase from that related to a pure time delay can be seen in Fig. 10(a)(ii), (b)(ii) and (c)(ii). This is due to the $10 \%$ difference in the natural frequencies of the shakers. The effect can also be seen as shift in the time delay at which the peak in the crosscorrelation coefficient occurs. Note that if the two shakers are identical, then this time shift does not occur. Also note, however, that it is highly unlikely for two shakers to be identical in practice.

From the simulations above it can be seen that the shaker dynamics can have an important effect on the cross-correlation function and time delay estimation, so it is particularly important to choose appropriate ones. As shown in Fig. 10, shakers that have a resonance frequency above the bandwidth of interest should be used. It is desirable for them to have resonance frequencies that are as high as possible, and this means that stiff actuators should be used. Such actuators are not electro-dynamic as used in the proof-of-concept prototype, but could be either magnetostrictive or piezoelectric. It is suggested that magnetostrictive actuators may be preferable as they can use readily available and inexpensive power amplifiers designed to drive an inductive load.

\section{Conclusions}

This paper has described the theoretical background and development of a proof-of-concept virtual pipe test-rig that can be used to simulate pipe vibration due to water leaks in the field. In principle, such a device could be used to compare the performances of different acoustic correlators for different pipelines. Using knowledge of fluid-filled pipe vibration, a proof-of-concept device has been built and tested, and was shown to perform reasonably well in the frequency range of $20-80 \mathrm{~Hz}$. This frequency range is suitable for typical plastic water distribution pipes and trunk mains. The limitation of the virtual pipe test-rig is the bandwidth, and this is due to the shakers. In the system described in this paper, simple, small loudspeakers with integrated power amplifiers were used as shakers. It has been shown that stiff actuators that have a high resonance frequency would be much more appropriate. 


\section{Acknowledgements}

The authors would like to acknowledge the financial support of the Coordenação de Aperfeiçoamento de Pessoal de Nível Superior do Ministério da Educação (CAPES) and the Fundação de Amparo à Pesquisa do Estado de São Paulo (FAPESP).

\section{References}

[1] Kingdom W, Liemberger R, Marin P. The challenge of reducing non-revenue water (NRW) in developing countries, how the private sector can help: a look at performance-based service contracting. Water Supply and Sanitation Sector Board Discussion Paper Series, Paper No. 8, December 2006.

[2] Fuchs HV, Riehle R. Ten years of experience with leak detection by acoustic signals analysis. Appl Acoust 1991;33:1-19.

[3] Hunaidi O, Chu WT. Acoustical characteristics of leak signals in plastic water distribution pipes. Appl Acoust 1999;58(3):235-54.

[4] Hunaidi O, Chu WT, Wang A, Guan W. Detecting leaks in plastic pipes. J Am Water Works Assoc 2000;92:82-94.

[5] Gao Y, Brennan MJ, Joseph PF, Muggleton JM, Hunaidi O. A model of the correlation function of leak noise in buried plastic pipes. J Sound Vib 2004;277: 133-48.

[6] Muggleton JM, Brennan MJ, Pinnington RJ. Wavenumber prediction of waves in buried pipes for water leak detection. J Sound Vib 2002;249:934-54.
77] Muggleton JM, Brennan MJ, Linford PW. Axisymmetric wave propagation in fluid-filled pipes: Measurements in in-vacuo and buried pipes. J Sound Vib 2004;270:171-90.

[8] Gao Y, Brennan MJ, Joseph PF, Muggleton JM, Hunaidi O. A model of the correlation of leak noise in buried plastic water pipes. J Sound Vib 2004;277: $133-48$.

[9] Gao Y, Brennan MJ, Joseph PF, Muggleton JM, Hunaidi O. On the selection of acoustic/vibration sensors for leak detection in plastic water pipes. J Sound Vib 2005;283:927-41.

[10] Gao Y, Brennan MJ, Joseph PF. A comparison of time delay estimators for the detection of leak-noise signals in buried plastic water distribution pipes. J Sound Vib 2006;292:552-70.

[11] Brennan MJ, Gao Y, Joseph PF. On the relationship between time and frequency domain methods in time for leak detection in plastic water pipes. J Sound Vib 2007;304(1-2):213-23.

[12] Almeida FCL, Brennan MJ, Joseph PF, Whitfield S, Dray S, Paschoalini A. On the acoustic filtering of the pipe and sensor in a buried plastic water pipe and its effect on leak detection: an experimental investigation. Sensors 2014;14: 5595-610.

[13] Almeida FCL. Improved acoustic methods for leak detection in buried plastic water distribution pipes [Ph.D. thesis]. University of Southampton: Southampton, UK; 2013.

[14] Almeida FCL, Brennan MJ, Joseph PF, Whitfield S, Dray S, Paschoalini A. Measurement of wave attenuation in buried plastic water distribution pipes. Strojniški vestnik - J Mech Eng 2014;60(5):298-306.

[15] Papastefanou AS, Joseph PF Brennan MJ. Experimental investigation into the characteristics of in-pipe leak noise in plastic water filled pipes. Acta Acust United Acust 2012;98(6):847-56. 